\title{
CUIDADOS A UN PACIENTE CON TRASTORNO DE ANSIEDAD GENERALIZADA
}

\section{SILVIA GARCÍA LIÑÁN ${ }^{1}$ Y VICENTE GALLARDO ORTIZ²}

${ }^{1}$ Graduada en enfermería. Universidad de Granada.

${ }^{2}$ Médico residente de medicina familiar y comunitaria. Hospital Comarcal de Baza (Granada).

\section{INTRODUCCIÓN}

El trastorno de ansiedad generalizada (TAG) es uno de los problemas del estado del ánimo más frecuentes y su síntoma principal es la preocupación crónica y persistente sobre gran número de hechos y actividades de la vida diaria ${ }^{1}$. Los criterios diagnósticos descritos en el Manual diagnóstico y estadístico de los trastornos mentales (DSM-5) incluyen preocupación excesiva con una duración de al menos seis meses, relacionado con al menos otros tres síntomas como inquietud o impaciencia, tensión muscular, alteraciones del sueño, irritabilidad, dificultad de concentración y fatiga².

Los trastornos de ansiedad —y, en especial, el TAGson muy comunes en atención primaria, con una prevalencia anual entre el 3 y el $8 \%$. Además, presenta mayor predominio en personas de 40-50 años, siendo más frecuente en el sexo femenino $(2: 1)^{3}$.

A pesar de su frecuencia, solo es diagnosticado y tratado de manera adecuada en menos de un tercio de los pacientes. Como consecuencia de este tras-

Correspondencia: Silvia García Liñán Correo electrónico: silviag/9@hotmail.com torno, se advierte una alteración funcional y de la calidad de vida de los pacientes ${ }^{4}$.

El objetivo fue elaborar un plan de cuidados desde una visión integral de la paciente, encaminado a establecer los cuidados necesarios para conseguir una mejora de la calidad de vida de la paciente y su familia.

\section{EXPOSICIÓN DEL CASO CLÍNICO}

Mujer de 44 años, divorciada hace un año y madre de dos hijos, que acude a la consulta de atención primaria por encontrarse desde su separación constantemente nerviosa e irritable y no consigue controlar la situación. Conjuntamente, refiere cefalea, tensión muscular, sensación de ahogo ocasional, cansancio, dificultad para concentrarse e insomnio. Menciona empeoramiento en el último mes y que siente preocupación continua desde hace tiempo por la nueva situación de su vida, especialmente, por la familia, las finanzas y su salud.

No presenta antecedentes personales psiquiátricos ni enfermedades somáticas. Toma benzodiacepinas de forma irregular y analgésicos. 


\section{VALORACIÓN DE ENFERMERÍA}

Para ello, se utilizó la observación y la entrevista estructurada según el modelo de 11 patrones funcionales de Marjory Gordon ${ }^{5}$ (tabla 1 ).

\section{DIAGNÓSTICOS DE ENFERMERÍA Y PLANIFICACIÓN DE CUIDADOS}

Una vez realizada la recopilación de los datos, se usó la taxonomía NANDA ${ }^{6}$ (North American Nursing Diagnosis Association) para formular los diagnósticos enfermeros, se establecieron los resultados emplean- do la clasificación de resultados de enfermería $\mathrm{NOC}^{7}$ (Nursing Outcomes Classification) y se describieron las intervenciones y actividades enfermeras empleando la clasificación de intervenciones de enfermería $\mathrm{NIC}^{8}$ (Nursing Interventions Classification). Los indicadores de los resultados de cada diagnóstico se acompañan de tres mediciones (PV: puntuación en la valoración; PD: puntuación deseada y PA: puntuación alcanzada). Para evaluar los criterios de resultado, se utilizó una escala de tipo Likert que oscila de 1 (la peor puntuación posible) a 5 (la máxima puntuación alcanzable) (tabla 2).

\begin{tabular}{|c|c|}
\hline $\begin{array}{l}\text { 1. Percepción/manejo } \\
\text { de la salud }\end{array}$ & $\begin{array}{l}\text { NAMC. Sin antecedentes personales de interés. Toma benzodiacepinas } \\
\text { de forma irregular y analgésicos. } \\
\text { Hábitos tóxicos: fumadora de } 10 \text { cigarros/día. No presenta descuido } \\
\text { personal. }\end{array}$ \\
\hline 2. Nutricional/metabólico & $\begin{array}{l}\text { IMC: } 23,88 \mathrm{~kg} / \mathrm{m}^{2} \text {. Normopeso. Apetito aumentado. Conocimientos } \\
\text { sobre alimentación y dieta equilibrada adecuados. }\end{array}$ \\
\hline 3. Eliminación & Sin alteraciones. \\
\hline 4. Actividad/ejercicio & $\begin{array}{l}\text { Independiente para las ABVD. Comportamiento motor: inquietud y } \\
\text { nerviosismo. Refiere tensión muscular y sensación de ahogo ocasional. }\end{array}$ \\
\hline 5. Sueño/descanso & $\begin{array}{l}\text { Insomnio de conciliación y cansancio. Somnolencia diurna e } \\
\text { irritabilidad. Uso de hipnóticos. }\end{array}$ \\
\hline 6. Cognitivo/perceptivo & $\begin{array}{l}\text { Consciente y orientada en las tres esferas. Contenido del pensamiento } \\
\text { coherente y organizado. Curso del pensamiento normal. Dificultad para } \\
\text { concentrarse. }\end{array}$ \\
\hline 7. Autopercepción/autoconcepto & $\begin{array}{l}\text { Ansiedad. Constantemente preocupada e inquieta. Refiere cefalea y } \\
\text { agotamiento emocional. }\end{array}$ \\
\hline 8. Rol/relaciones & Vive con sus dos hijos de 8 y 10 años. Divorciada hace un año. \\
\hline 9. Sexualidad/reproducción & Sin pareja actual. \\
\hline $\begin{array}{l}\text { 10. Adaptación/tolerancia } \\
\text { al estrés }\end{array}$ & $\begin{array}{l}\text { Continuamente nerviosa, irritable y preocupada. No consigue controlar } \\
\text { la situación. }\end{array}$ \\
\hline 11. Valores/creencias & Sin alteraciones. \\
\hline
\end{tabular}

ABVD: actividades básicas de la vida diaria; IMC: índice de masa corporal; NAMC: no alergias medicamentosas conocidas. 
Tabla 2. Diagnósticos (NANDA), objetivos (NOC) e intervenciones (NIC) de enfermería

\begin{tabular}{|c|c|}
\hline NANDA & $\begin{array}{l}\text { (00146) Ansiedad r/c cambio en: la situación económica, el estado de salud, la función del rol, } \\
\text { asociación familiar m/p insomnio, inquietud, irritabilidad, nerviosismo, preocupación creciente, } \\
\text { dificultad para la concentración, dificultades respiratorias. }\end{array}$ \\
\hline NOC & $\begin{array}{l}1211 \text { Nivel de ansiedad. Indicadores: } \\
\text { - } 121105 \text { Inquietud. } \\
\text { - } 121108 \text { Irritabilidad. } \\
\text { - } 121106 \text { Tensión muscular. }\end{array}$ \\
\hline NIC & $\begin{array}{l}5820 \text { Disminución de la ansiedad. Actividades: } \\
\text { - Animar la manifestación de sentimientos, percepciones y miedos. } \\
\text { - Identificar los cambios en el nivel de ansiedad. } \\
\text { - Establecer actividades recreativas encaminadas a la reducción de tensiones. }\end{array}$ \\
\hline NANDA & $\begin{array}{l}\text { (00069) Afrontamiento ineficaz r/c crisis situacionales, incertidumbre, falta de confianza en la } \\
\text { capacidad para afrontar la situación m/p fatiga, dificultad de concentración, trastornos del } \\
\text { patrón del sueño. }\end{array}$ \\
\hline NOC & $\begin{array}{l}1302 \text { Afrontamiento de problemas. Indicadores: } \\
\text { - } 130204 \text { Refiere disminución de estrés. } \\
\text { - } 130212 \text { Utiliza estrategias de superación efectivas. }\end{array}$ \\
\hline NIC & $\begin{array}{l}5240 \text { Asesoramiento. Actividades: } \\
\text { - Ayudar al paciente a identificar el problema o la situación causante del trastorno. } \\
\text { - Practicar técnicas de reflexión y clarificación para facilitar la expresión de preocupaciones. } \\
5270 \text { Apoyo emocional. Actividades: } \\
\text { - Escuchar las expresiones de sentimientos y creencias. } \\
\text { - Ayudar al paciente a reconocer sentimientos tales como la ansiedad, ira o tristeza. }\end{array}$ \\
\hline NANDA & $\begin{array}{l}\text { (00095) Insomnio r/c ansiedad, estrés m/p expresa falta de energía, cambios de humor, } \\
\text { dificultad para concentrarse, dificultad para conciliar el sueño. }\end{array}$ \\
\hline NOC & $\begin{array}{l}0004 \text { Sueño. Indicadores: } \\
\text { - } 000401 \text { Horas de sueño. } \\
\text { - } 000404 \text { Calidad del sueño. }\end{array}$ \\
\hline NIC & $\begin{array}{l}6040 \text { Terapia de relación simple. Actividades: } \\
\text { - Explicar el fundamento de la relajación y sus beneficios, límites y tipos de relajación } \\
\text { disponibles. } \\
\text { - Inducir conductas que estén condicionadas para producir relajación, como respiración } \\
\text { profunda, bostezos, respiración abdominal e imágenes de paz. }\end{array}$ \\
\hline
\end{tabular}

m/p: manifestado por; NANDA: diagnósticos enfermeros (North American Nursing Diagnosis Association); NIC: intervenciones enfermeras (Nursing Interventions Classification); NOC: criterios de resultados de enfermería (Nursing Outcomes Classification); PA: puntuación alcanzada; PD: puntuación deseada; PV: puntuación en la valoración; r/c: relacionado con.

\section{DISCUSIÓN Y CONCLUSIONES}

EI TAG es un trastorno de ansiedad prevalente que se observa especialmente en la población femenina y genera un nivel considerable de disfunción. Además, con frecuencia se asocia a comorbilidad somática y psiquiátrica y aumento del riesgo de enferme- 
dades como la hipertensión y los trastornos digestivos o cardiovasculares ${ }^{1,3}$.

A pesar de su frecuencia, solo es diagnosticado y tratado de manera adecuada en menos de un tercio de los pacientes. Como consecuencia del TAG, se aprecia una alteración funcional y de la calidad de vida, además de un coste notable por la utilización de los servicios de salud ${ }^{4}$. Un estudio estimó que el promedio de costes directos anuales por paciente en atención primaria fueron de 817,37 €9.

El grado de resolución del caso ha sido satisfactorio, ya que hemos conseguido: disminuir el nivel de ansiedad a través de la manifestación de sus sentimientos, percepciones y miedos y la realización de actividades recreativas encaminadas a la reducción de tensiones; la utilización por parte de la paciente de estrategias efectivas de afrontamiento de problemas mediante asesoramiento y apoyo emocional y mejora del sueño empleando terapias de relajación simple.

Para concluir, dado que son situaciones puntuales, pero que generan un gran sufrimiento y tienen tendencia a la repetición, es primordial una atención integral y multidisciplinaria temprana por parte de los equipos de salud, centrada en la detección precoz, el tratamiento sintomático adecuado y el apoyo psicológico y social para prevenir la comorbilidad asociada y aumentar la calidad de vida de estos pacientes.

\section{BIBLIOGRAFÍA}

1. Langarita-Llorente R, Gracia-García P. Neuropsicología del trastorno de ansiedad generalizada: revisión sistemática. Rev Neurol. 2019;69(2):59-67.

2. American Psychiatric Association. DSM-5: manual diagnóstico y estadístico de los trastornos mentales. Madrid: Editorial Médica Panamericana; 2018.

3. Amor Mercado G, Vega Núñez A, Villena Jimena A, Gómez Ocaña C, Morales-Asencio JM, Hurtado Lara MM. Implicación de usuarios diagnosticados de trastorno de ansiedad generalizada en la elaboración de una guía de práctica clínica. Universitas Psychologica. 2018;17(2).

4. Katzman MA, Bleau P, Blier P, Chokka P, Kjernisted K, Van Ameringen $\mathrm{M}$, et al. Canadian clinical practice guidelines for the management of anxiety, posttraumatic stress and obsessive-compulsive disorders. BMC Psychiatry. 2014;14 Suppl 1(Suppl 1):S1.

5. Gordon M. Manual de diagnósticos enfermeros. 11. ${ }^{a}$ ed. Madrid: McGraw-Hill Interamericana de España S.L.; 2007.

6. NANDA International. Herdman TH, Kamitsuru S. Diagnósticos enfermeros. Definiciones y clasificación 20152017. 10. a ed. Barcelona: Elsevier España, S.L.; 2015.

7. Morrhead S, Johnson M, Mass M, Swanson E. Clasificación de resultados de enfermería (NOC). Medición de resultados en salud. 5. ${ }^{a}$ ed. Barcelona: Elsevier España, S.L.; 2014

8. Bulecheck GM, Butcher HK, Dochterman JC, Wagner $\mathrm{CM}$. Clasificación de intervenciones de enfermería (NIC). 6. ${ }^{a}$ ed. Barcelona: Elsevier España, S.L.; 2014.

9. Sicras-Mainar A, Blanca-Tamayo M, Navarro-Artieda R, Rejas-Gutiérrez J. Use of resources and costs profile in patients with fibromyalgia or generalized anxiety disorder in primary care settings. Aten Primaria. 2009;41(2): 77-84. 